\title{
Perfect bipolar fuzzy graphs
}

\author{
Basheer Ahamed Mohideen \\ Department of Mathematics, Faculty of Science, University of Tabuk, P. O. Box 741, Tabuk, 71491, Kingdom of Saudi Arabia.
}

\begin{abstract}
This paper introduces the concepts of perfect bipolar fuzzy graph, complete perfect bipolar fuzzy graph, semi-perfect bipolar fuzzy graph, and further investigates some of their properties.
\end{abstract}

Keywords: Bipolar fuzzy set, bipolar fuzzy graph, perfect fuzzy graph, perfect bipolar fuzzy graph, complete perfect bipolar fuzzy graph.

2010 MSC: 03E72, 05C99.

(C)2019 All rights reserved.

\section{Introduction}

The framework of fuzzy sets deals with imprecision in knowledge representation and information processing. The bipolar fuzzy set deals with the bipolarity of information, which occurs in some domains, such as preference modeling under some constraints, spatial reasoning, argumentation, etc.. In these domains, two types of information have often to be handled: (i) positive information (e.g., what is possible or desired) and (ii) negative information (e.g., rules, constraints). Zadeh [14] introduced the notion of fuzzy sets. Zhang [16] introduced the concept of bipolar fuzzy sets; it gives more precision, flexibility, and compatibility to the system as compared to the classical and fuzzy models.

Rosenfeld [13] discussed the concept of fuzzy graphs and obtained analogous of several graph theoretical concepts. Bhattacharya [8] gave some remarks on fuzzy graphs. Bhutani et al. [9] discussed strong arc in fuzzy graphs. Basheer et al. [6] introduced the concept of perfect fuzzy graphs. Basheer et al. [7] introduced the concept of closed neighborhood degree and its extension in fuzzy graphs. Akram [1] introduced bipolar fuzzy graphs, Akram et al. [3] studied regular bipolar fuzzy graphs, and Akram [2] discussed further notions and results in bipolar fuzzy graphs with applications. Basheer [5] introduced a semi-regular bipolar fuzzy graph, a semi-complete bipolar fuzzy graph, and discussed some types of degrees in bipolar fuzzy graph. This paper introduces the concepts of perfect bipolar fuzzy graph, complete perfect bipolar fuzzy graph, semi-perfect bipolar fuzzy graph, and investigates some of their properties.

Email address: bahamed@ut.edu.sa (Basheer Ahamed Mohideen)

doi: $10.22436 /$ jmcs.019.02.06

Received: 2018-11-17 Revised: 2019-04-03 Accepted: 2019-04-10 


\section{Preliminaries}

In this section, let us recall some basic notions and results, which are available in references [1-16].

\subsection{Fuzzy set and bipolar fuzzy set}

Definition 2.1. A fuzzy set (class) $A$ in $X$ is characterized by a membership (characteristic function) $f_{A}(X)$ which associates with each point in $X$ a real number in the interval $[0,1]$, with the value of $f_{A}(x)$ at $x$ representing the "grade of membership" of $x$ in $A$.

Definition 2.2. Let $V$ and $W$ be any two sets, and let $\mu$ and $\gamma$ be fuzzy subsets of $V$ and $W$, respectively. A fuzzy relation $\rho$ from the fuzzy subset $\mu$ into the fuzzy subset $\gamma$ is a fuzzy subset of $V \times W$ such that $\rho(v, w) \leqslant \mu(v) \wedge \gamma(w), \forall v \in V, w \in W$.

Definition 2.3. Let $X$ be a nonempty set. A bipolar fuzzy (sub)set $B$ in $X$ is an object having the form $B=\left\{x, \mu^{P}(x), \mu^{N}(x): x \in X\right\}$, where $\mu^{P}: X \rightarrow[0,1]$ and $\mu^{N}: X \rightarrow[-1,0]$ are mappings.

The positive membership degree $\mu^{\mathrm{P}}(x)$ is used to denote the satisfaction degree of an element $x$ to the property corresponding to a bipolar fuzzy set $B$, and the negative membership degree $\mu^{N}(x)$ to denote the satisfaction degree of an element $x$ to some implicit counter-property corresponding to a bipolar fuzzy set $B$. If $\mu^{\mathrm{P}}(x) \neq 0$ and $\mu^{\mathrm{N}}(x)=0$, it is the situation that $x$ is regarded as having only positive satisfaction for $B$. If $\mu^{\mathrm{P}}(\mathrm{x})=0$ and $\mu^{\mathrm{N}}(\mathrm{x}) \neq 0$, it is the situation that $\mathrm{x}$ does not satisfy the property of $\mathrm{B}$, but somewhat satisfies the counter property of $B$. It is possible for an element $x$ to be such $\mu^{P}(x) \neq 0, \mu^{N}(x) \neq 0$, when the membership function of the property overlaps that of its counter property over some portion of $X$. For the sake of simplicity, the symbol $B=\left(\mu^{P}, \mu^{N}\right)$ is used for the bipolar fuzzy set $B=\left\{x, \mu^{P}(x), \mu^{N}(x): x \in X\right\}$.

Definition 2.4. Let $X$ be a nonempty set. A mapping $A=\left(\mu_{A}^{P}, \mu_{A}^{N}\right): X \times X \rightarrow[-1,1] \times[-1,1]$ a bipolar fuzzy relation on $X$ such that $\mu^{P}(x, y) \in[0,1]$ and $\mu^{N}(x, y) \in[-1,0]$.

\subsection{Basic notions in graph theory}

Let us recall some basic definitions in graph theory [9], a graph is an ordered pair $G^{*}=(V, E)$, where $V$ is the nonempty set of vertices of $G^{*}$ and $E$ is the set of edges of $G^{*}$. Two vertices $x$ and $y$ in an undirected graph $\mathrm{G}^{*}$ are said to be adjacent (or neighbors) in $\mathrm{G}^{*}$ if $\{x, y\}$ is an edge of $\mathrm{G}^{*}$. A simple graph is an undirected graph that has no loops and/or no parallel edges. The neighborhood of a vertex $v$ in a graph $\mathrm{G}^{*}$ is the induced subgraph of $\mathrm{G}^{*}$ consisting of all vertices adjacent to $v$ and all edges connecting two such vertices. The neighborhood is often denoted by $N(v)$. The degree $\operatorname{deg}(x)$ of vertex $x$ ( $\operatorname{simply}$ $d(x))$ is the number of edges incident on $x$ or equivalently, $\operatorname{deg}(x)=|N(x)|$. The set of neighbors, called a (open) neighborhood $\mathrm{N}(x)$ for a vertex $x$ in a graph $\mathrm{G}^{*}$, consists of all vertices adjacent to $x$ but not including $x$, that is, $N(x)=\{y \in V: x y \in E\}$. When $x$ is included, it is called a closed neighborhood, denoted by $N[x]$, that is, $N[x]=N(x) \cup\{x\}$. A regular graph is a graph where each vertex has the same number of neighbors, that is, all the vertices have the same open neighborhood degree. A complete graph is a simple graph in which every pair of distinct vertices is connected by an edge.

\subsection{Basic notions in fuzzy graphs}

A fuzzy graph $G=(V, \mu, \rho)$ is a nonempty set $V$ together with a pair of functions $\mu: V \rightarrow[0,1]$ and $\rho: V \times V \rightarrow[0,1]$ such that $\rho(x, y) \leqslant \mu(x) \wedge \mu(y)$, for all $x, y \in V$, where $\mu(x) \wedge \mu(y)$ denotes the minimum of $\mu(x)$ and $\mu(y)$. A fuzzy graph $G$ is called strong if $\rho(x, y)=\mu(x) \wedge \mu(y), \forall\{x, y\} \in V \times V$. A fuzzy graph $G$ is called complete if $\rho(x, y)=\mu(x) \wedge \mu(y), \forall x, y \in V$. Note that if $\rho(x, y)>0$, then we call $x$ and $y$ neighbors. The order and size of a fuzzy graph $G$ are defined as $O(G)=\sum_{x \in V} \mu(x)$ and $S(G)=\sum_{\{x, y\} \in E} \rho(\{x, y\})$, respectively. An edge $\{x, y\}$ of a fuzzy graph $G$ is called an effective edge if $\rho(\{x, y\})=\mu(x) \wedge \mu(y)\} . N(x)=\{y \in V: \rho(\{x, y\})=\mu(x) \wedge \mu(y)\}$ is called the neighborhood of $x$, and $N[x]=N(x) \cup\{x\}$ is called the closed neighborhood of $x$. The degree of a vertex can be categorized in different ways for a fuzzy graph. The effective degree of a vertex $x$ is defined as the sum 
of the membership value of the effective edges incident with $x$, that is, $d_{E}(x)=\sum_{\{x, y\} \in E} \rho_{E}(\{x, y\})$. The neighborhood degree of a vertex is defined as the sum of the membership value of the neighborhood vertices of $x$, and is denoted by $d_{N}(x)$.

\subsection{Bipolar fuzzy graphs}

Definition 2.5. A bipolar fuzzy graph with an underlying set $V$ is defined to be a pair $G=(A, B)$, where $A=\left(\mu_{A}^{P}, \mu_{A}^{N}\right)$ is a bipolar fuzzy set in $V$ and $B=\left(\mu_{B}^{P}, \mu_{B}^{N}\right)$ is a bipolar fuzzy set in $E \subseteq V \times V$ such that $\mu_{B}^{P}(\{x, y\}) \leqslant \min \left(\mu_{A}^{P}(x), \mu_{A}^{P}(y)\right)$ and $\mu_{B}^{N}(\{x, y\}) \geqslant \max \left(\mu_{A}^{N}(x), \mu_{A}^{N}(y)\right), \forall x, y \in V$. Here, $A$ denotes the bipolar fuzzy vertex set of $\mathrm{V}$ and $\mathrm{B}$ denotes the bipolar fuzzy edge set of $E$.

Note that $B$ is symmetric bipolar fuzzy relation on $A$. We use notion $x y$ for an element of $E$. Thus $G=(A, B)$ is a bipolar fuzzy graph of $G^{*}=(V, E)$ if $\mu_{B}^{P}(x y) \leqslant \min \left(\mu_{A}^{P}(x), \mu_{A}^{P}(y)\right)$ and $\mu_{B}^{N}(x y) \geqslant$ $\max \left(\mu_{A}^{N}(x), \mu_{A}^{N}(y)\right)$ for all $x, y \in V, x y \in E$.

In other words, the Definition 2.5 can be written as, a bipolar fuzzy graph $G=(V, E, \mu, \rho)$, where $V$ is a nonempty vertex set, $\mathrm{E} \subseteq \mathrm{V} \times \mathrm{V}$ is an edge set, $\mu$ is bipolar fuzzy set in $\mathrm{V}$, and $\rho$ is bipolar fuzzy set in $\mathrm{E}$, that is, $\mu(x)=\left(\mu^{P}(x), \mu^{N}(x)\right)$ and $\rho(x y)=\left(\rho^{P}(x y), \rho^{N}(x y)\right)$ such that $\rho^{P}(x y) \leqslant \min \left(\mu^{P}(x), \mu^{P}(y)\right)$ and $\rho^{N}(x y) \geqslant \max \left(\mu^{N}(x), \mu^{N}(y)\right), \forall x, y \in V, x y \in E$. Here, $x y$ means that $\{x, y\}$, an undirected edge. The notation $G=(V, E, \mu, \rho)$ is used for bipolar fuzzy graph, where $V$ is a nonempty vertex set, $E$ is an edge set, is fuzzy subset of $V$, and $\rho$ is fuzzy subset of $E$.

Definition 2.6. A bipolar fuzzy graph $G=(V, E, \mu, \rho)$ is said to be strong bipolar fuzzy graph if $\rho^{\mathrm{P}}(x y)=$ $\min \left(\mu^{\mathrm{P}}(\mathrm{x}), \mu^{\mathrm{P}}(\mathrm{y})\right)$ and $\rho^{\mathrm{N}}(x y)=\max \left(\mu^{\mathrm{N}}(\mathrm{x}), \mu^{\mathrm{N}}(\mathrm{y})\right), \forall x y \in \mathrm{E}$.

Definition 2.7. A bipolar fuzzy graph $G=(V, E, \mu, \rho)$ is said to be complete bipolar fuzzy graph if $\rho^{P}(x y)=$ $\min \left(\mu^{\mathrm{P}}(\mathrm{x}), \mu^{\mathrm{P}}(\mathrm{y})\right)$ and $\rho^{\mathrm{N}}(\mathrm{xy})=\max \left(\mu^{\mathrm{N}}(\mathrm{x}), \mu^{\mathrm{N}}(\mathrm{y})\right), \forall x, y \in \mathrm{V}$.

Definition 2.8. LetG $=(V, E, \mu, \rho)$ be a bipolar fuzzy graph. The neighborhood of a vertex $x \in G$ is defined by $N(x)=\left(N_{\mu}(x), N_{\gamma}(x)\right)$, where $N_{\mu}(x)=\left\{y \in V: \mu_{B}^{P}(x y) \leqslant \min \left(\mu_{A}^{P}(x), \mu_{A}^{P}(y)\right)\right\}$ and $N_{\gamma}(x)=$ $\left\{y \in V: \mu_{B}^{N}(x y) \geqslant \max \left(\mu_{A}^{N}(x), \mu_{A}^{N}(y)\right\}\right.$.

Definition 2.9. Let $\mathrm{G}$ be a bipolar fuzzy graph. The (open) neighborhood degree of a vertex $x$ in $\mathrm{G}$ is defined as $\operatorname{deg}(x)=\left(\operatorname{deg}_{\mu}(x), \operatorname{deg}_{\gamma}(x)\right)$, where $\operatorname{deg}_{\mu}(x)=\sum_{y \in N(x)} \mu_{A}^{P}(y), \operatorname{deg}_{\gamma}(x)=\sum_{y \in N(x)} \mu_{A}^{N}(y)$.

Note that $\mu_{\mathrm{B}}^{\mathrm{P}}(x y)>0, \mu_{\mathrm{B}}^{\mathrm{N}}(x y)<0$ for $x y \in E ; \mu_{\mathrm{B}}^{\mathrm{P}}(x y)=\mu_{\mathrm{B}}^{\mathrm{N}}(x y)=0$ for $x y \notin E$.

Definition 2.10. Let $\mathrm{G}$ be a bipolar fuzzy graph. The closed neighborhood degree of a vertex $x$ in $\mathrm{G}$ is defined

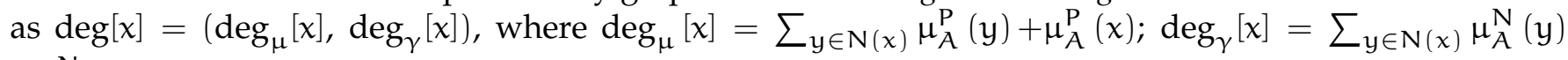
$+\mu_{A}^{N}(x)$.

Definition 2.11. A bipolar fuzzy graph $G=(V, E, \mu, \rho)$ is called $\left(k_{1}, k_{2}\right)$-regular if $d(v)=\left(k_{1}, k_{2}\right)$, where $\mathrm{k}_{1}=\mathrm{d}^{\mathrm{P}}(v)$ and $\mathrm{k}_{2}=\mathrm{d}^{\mathrm{N}}(v)$.

Hereafter, the neighborhood degree of a vertex $v$ is denoted by $d_{N}(v)$ and the closed neighborhood degree of a vertex $v$ is denoted by $d_{N}[v]$.

The order of bipolar fuzzy graph is a pair of positive order and negative order of bipolar fuzzy graph and the size of bipolar fuzzy graph is a pair of positive size and negative size of bipolar fuzzy graph.

Definition 2.12. Let $G=(V, E, \mu, \rho)$ be a bipolar fuzzy graph. The order of bipolar fuzzy graph, denoted by $\mathrm{O}(\mathrm{G})$, is defined as $\mathrm{O}(\mathrm{G})=\left(\mathrm{O}^{\mathrm{P}}(\mathrm{G}), \mathrm{O}^{\mathrm{N}}(\mathrm{G})\right)$, where $\mathrm{O}^{\mathrm{P}}(\mathrm{G})=\sum_{v \in V} \mu^{\mathrm{P}}(v) ; \mathrm{O}^{\mathrm{N}}(\mathrm{G})=\sum_{v \in V} \mu^{\mathrm{N}}(v)$. Similarly, the size of bipolar fuzzy graph, denoted by $S(G)$, is defined as $S(G)=\left(S^{P}(G), S^{N}(G)\right)$, where $S^{P}(G)=\sum_{v w \in E} P(v w) ; S^{N}(G)=\sum_{\nu w \in E} N(v w)$.

Proposition 2.13. In a bipolar fuzzy graph $\mathrm{G}=(\mathrm{V}, \mathrm{E}, \mu, \rho)$, the following inequalities hold: (i) $\mathrm{O}^{\mathrm{P}}(\mathrm{G}) \geqslant \mathrm{S}^{\mathrm{P}}(\mathrm{G})$; (ii) $\mathrm{O}^{\mathrm{N}}(\mathrm{G}) \leqslant \mathrm{S}^{\mathrm{N}}(\mathrm{G})$. 
Corollary 2.14. In a regular bipolar fuzzy graph $\mathrm{G}=(\mathrm{V}, \mathrm{E}, \mu, \rho)$, the following inequalities hold: (i) $\mathrm{O}^{\mathrm{P}}(\mathrm{G})=$ $\mathrm{S}^{\mathrm{P}}(\mathrm{G}) ;\left(\right.$ ii) $\mathrm{O}^{\mathrm{N}}(\mathrm{G})=\mathrm{S}^{\mathrm{N}}(\mathrm{G})$.

Definition 2.15. Let $G=(V, E, \mu, \rho)$ be a bipolar fuzzy graph. An edge is called effective if $\rho^{\mathrm{P}}(\nu w)=$ $\min \left\{\mu^{\mathrm{P}}(v), \mu^{\mathrm{P}}(w)\right\}$ and $\rho^{\mathrm{N}}(v w)=\max \left\{\mu^{\mathrm{N}}(v), \mu^{\mathrm{N}}(w)\right\}$ for all $x y \in E$. It is denoted by $\rho_{\mathrm{E}}(\nu w)=$ $\left(\rho_{\mathrm{E}}^{\mathrm{P}}(\nu w), \rho_{\mathrm{E}}^{\mathrm{N}}(\nu w)\right)$. The effective degree of a vertex $\nu$ in bipolar fuzzy graph $\mathrm{G}$, denoted by $\mathrm{d}_{\mathrm{E}}(\nu)$, is defined as $d_{E}(v)=\sum_{\nu w \in E}\left(\rho_{E}^{P}(\nu w), \rho_{E}^{N}(v w)\right)$.

Definition 2.16. Let $G=(V, E, \mu, \rho)$ be a bipolar fuzzy graph. The ordinary degree (simply degree) of a vertex $v$ in bipolar fuzzy graph $G$, denoted by $d(v)$, is defined as $d(v)=\sum_{v w \in E}\left(\rho^{P}(\nu w), \rho^{N}(v w)\right)$.

Note that $d_{E}\left(v_{3}\right)=(0,0)$ means that there is no effective edge incidence with $v_{3}$.

Definition 2.17. A bipolar fuzzy graph $G=(V, E, \mu, \rho)$ is said to be semi-regular if all vertices have equal closed neighborhood degrees. We say that $G$ is $\left(k_{1}, k_{2}\right)$-semi-regular if $d_{N}[v]=\left(k_{1}, k_{2}\right), \forall v \in V$, and $k_{1}, k_{2} \in \mathbb{R}$, where $\mathbb{R}$ is real number set.

Definition 2.18. A bipolar fuzzy graph $G=(V, E, \mu, \rho)$ is said to be semi-complete if it satisfies (crisp) complete graph condition, but $\mathrm{G}$ does not satisfy strong bipolar fuzzy graph condition.

Proposition 2.19. In a strong bipolar fuzzy graph $\mathrm{G}=(\mathrm{V}, \mathrm{E}, \mu, \rho), \mathrm{d}(v)=\mathrm{d}_{\mathrm{E}}(v), \forall v \in \mathrm{V}$.

Proposition 2.20. Every complete bipolar fuzzy graph is semi-regular bipolar fuzzy graph.

Note that every complete bipolar fuzzy graph does not need to be regular bipolar fuzzy graph.

Proposition 2.21. In any complete bipolar fuzzy graph $\mathrm{G}, \mathrm{d}_{\mathrm{N}}[v]=\mathrm{O}(\mathrm{G}), \forall v \in \mathrm{V}$. That is, the closed neighborhood degree of any vertex is equal to the order of bipolar fuzzy graph.

Proposition 2.22. In a regular bipolar fuzzy graph $\mathrm{G}=(\mathrm{V}, \mathrm{E}, \mu, \rho)$, closed neighborhood degree, order of bipolar fuzzy graph and size of bipolar fuzzy graph are equal.

Proposition 2.23. In a regular bipolar fuzzy graph $\mathrm{G}=(\mathrm{V}, \mathrm{E}, \mu, \rho)$, ordinary degrees, effective degrees and neighborhood degrees are equal for any vertex in $G$. That is, $\mathrm{d}(v)=\mathrm{d}_{\mathrm{E}}(v)=\mathrm{d}_{\mathrm{N}}(v), \forall v \in \mathrm{V}$.

Proposition 2.24. Every complete bipolar fuzzy graph is strong bipolar fuzzy graph.

Note that the converse of Proposition 2.24 need not be true.

Proposition 2.25. Every semi-complete bipolar fuzzy graph is semi-regular bipolar fuzzy graph.

Note that the converse of Proposition 2.25 is also true.

\section{Perfect bipolar fuzzy graphs}

In constructing a bipolar fuzzy graph network in which the bipolar or double-sided (positive and negative, or effect and side effect) nature of human perception occurs, we need to consider both extreme values (positive extreme 1 and negative extreme -1) and develop a concept of perfectness in bipolar fuzzy graphs. In this situation, we have to analyze the perfectness of each vertex (point/junction/node) and each edge (line/route/path) independently. This section introduces the concepts of perfect bipolar fuzzy graph, complete perfect bipolar fuzzy graph, semi-perfect bipolar fuzzy graph, and investigates some results.

In this article, we consider $G=(V, E, \mu, \rho)$ is always a connected undirected simple bipolar fuzzy graph.

Definition 3.1. A vertex $v$ in a bipolar fuzzy graph $G=(V, E, \mu, \rho)$ is called a perfect bipolar fuzzy vertex if $\mu^{\mathrm{P}}(v)=1$ and $\mu^{\mathrm{N}}(v)=-1$, i.e., $\mu(v)=(1,-1), \forall v \in \mathrm{V}$. 
Definition 3.2. An edge $e=\{v, w\}$ (simply $v w$ ) in a bipolar fuzzy graph $G=(V, E, \mu, \rho)$ is called a perfect bipolar fuzzy edge if $\rho^{\mathrm{P}}(v w)=1$ and $\rho^{\mathrm{N}}(\nu w)=-1$, (i.e., $) \rho(\nu w)=(1,-1), \forall v w \in \mathrm{E}$.

Example 3.3. Let $G=(V, E, \mu, \rho)$ be a bipolar fuzzy graph, where $V=\left\{v_{1}, v_{2}, v_{3}, v_{4}\right\}, E=\left\{v_{1} v_{2}, v_{1} v_{4}\right.$, $\left.v_{2} v_{3}, v_{2} v_{4}, v_{3} v_{4}\right\}$ with $\mu\left(v_{1}\right)=(0.9,-1), \mu\left(v_{2}\right)=(1,-0.8), \mu\left(v_{3}\right)=(1,-1), \mu\left(v_{4}\right)=(0.5,-0.5) ; \rho\left(v_{1} v_{2}\right)=$ $(0.8,-0.7), \rho\left(v_{1} v_{4}\right)=(0.3,-0.3), \rho\left(v_{2} v_{3}\right)=(1,-0.8), \rho\left(v_{2} v_{4}\right)=(0.4,-0.5), \rho\left(v_{3} v_{4}\right)=(0.5,-0.3)$. Here $v_{3}$ is the only perfect bipolar fuzzy vertex; but no perfect bipolar fuzzy edge.

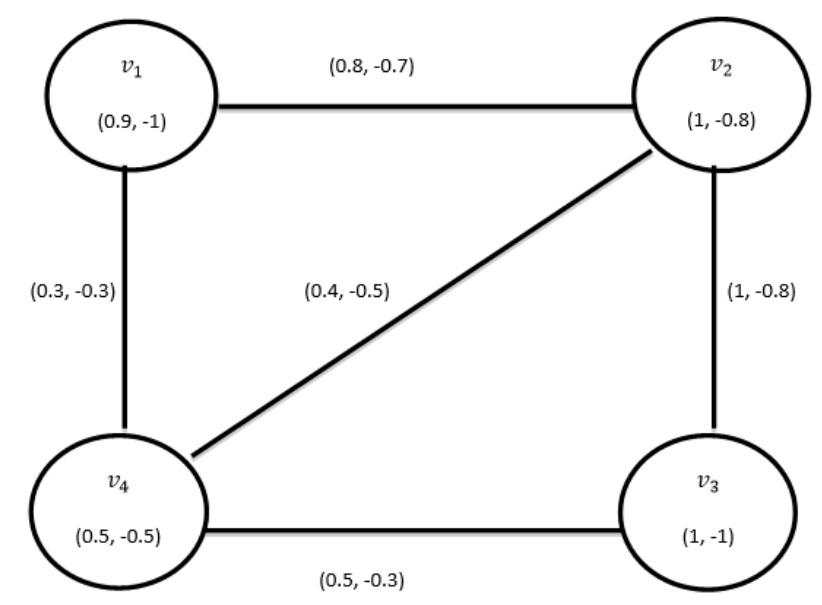

Figure 1: Example 3.3

Example 3.4. Let $G=(V, E, \mu, \rho)$ be a bipolar fuzzy graph, where $V=\left\{v_{1}, v_{2}, v_{3}, v_{4}\right\}, E=\left\{v_{1} v_{2}, v_{1} v_{4}\right.$, $\left.\nu_{2} \nu_{3}, \nu_{2} v_{4}, \nu_{3} v_{4}\right\}$ with $\mu\left(v_{1}\right)=(0.9,-1), \mu\left(v_{2}\right)=(1,-1), \mu\left(v_{3}\right)=(1,-1), \mu\left(v_{4}\right)=(0.5,-0.5) ; \rho\left(\nu_{1} v_{2}\right)=$ $(0.8,-0.7), \rho\left(v_{1} v_{4}\right)=(0.3,-0.3), \rho\left(v_{2} v_{3}\right)=(1,-1), \rho\left(v_{2} v_{4}\right)=(0.4,-0.5), \rho\left(v_{3} v_{4}\right)=(0.5,-0.3)$. Here $v_{2}, v_{3}$ are perfect bipolar fuzzy vertices; $v_{2} v_{3}$ is the only perfect bipolar fuzzy edge.

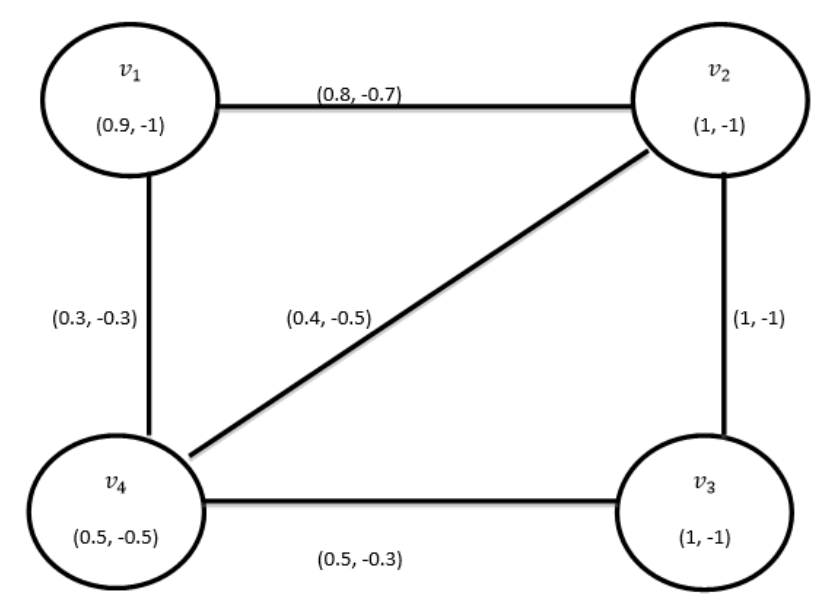

Figure 2: Example 3.4

Definition 3.5. A bipolar fuzzy graph $G=(V, E, \mu, \rho)$ is called an $\mu$-perfect bipolar fuzzy graph if all vertices in $\mathrm{G}$ are perfect bipolar fuzzy vertices.

Definition 3.6. A bipolar fuzzy graph $G=(V, E, \mu, \rho)$ is called an $\rho$-perfect bipolar fuzzy graph if all edges in $\mathrm{G}$ are perfect bipolar fuzzy edges. 
Example 3.7. Let $G=(V, E, \mu, \rho)$ be a bipolar fuzzy graph, where $V=\left\{v_{1}, v_{2}, v_{3}, v_{4}\right\}, E=\left\{v_{1} v_{2}, v_{1} v_{4}\right.$, $\left.v_{2} v_{3}, v_{2} v_{4}, v_{3} v_{4}\right\}$ with $\mu\left(v_{1}\right)=\mu\left(v_{2}\right)=\mu\left(v_{3}\right)=\mu\left(v_{4}\right)=(1,-1) ; \rho\left(v_{1} v_{2}\right)=(0.8,-0.7), \rho\left(v_{1} v_{4}\right)=(0.3,-0.3)$, $\rho\left(v_{2} v_{3}\right)=(1,-1), \rho\left(v_{2} v_{4}\right)=(0,4,-0.5), \rho\left(v_{3} v_{4}\right)=(0,5,-0.3)$. Here all vertices are perfect bipolar fuzzy vertices. Therefore, this is an $\mu$-perfect bipolar fuzzy graph. But, this is not an $\rho$-perfect bipolar fuzzy graph.

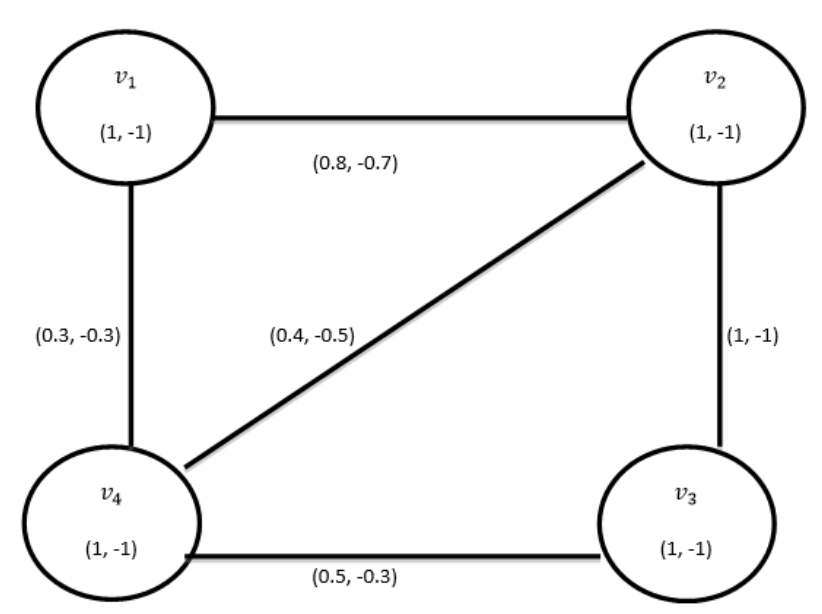

Figure 3: $\mu$-perfect bipolar fuzzy graph.

Example 3.8. Let $G=(V, E, \mu, \rho)$ be a bipolar fuzzy graph, where $V=\left\{v_{1}, v_{2}, v_{3}, v_{4}\right\}, E=\left\{v_{1} v_{2}, v_{1} v_{4}\right.$, $\left.v_{2} v_{3}, v_{2} v_{4}, v_{3} v_{4}\right\}$ with $\mu\left(v_{1}\right)=\mu\left(v_{2}\right)=\mu\left(v_{3}\right)=\mu\left(v_{4}\right)=(1,-1) ; \rho\left(v_{1} v_{2}\right)=(1,-1), \rho\left(v_{1} v_{4}\right)=(1,-1)$, $\rho\left(v_{2} v_{3}\right)=(1,-1), \rho\left(v_{2} v_{4}\right)=(1,-1), \rho\left(v_{3} v_{4}\right)=(1,-1)$. Here all vertices are perfect bipolar fuzzy vertices and all edges are bipolar fuzzy edges. Therefore, this is an $\mu$-perfect and $\rho$-perfect bipolar fuzzy graph.

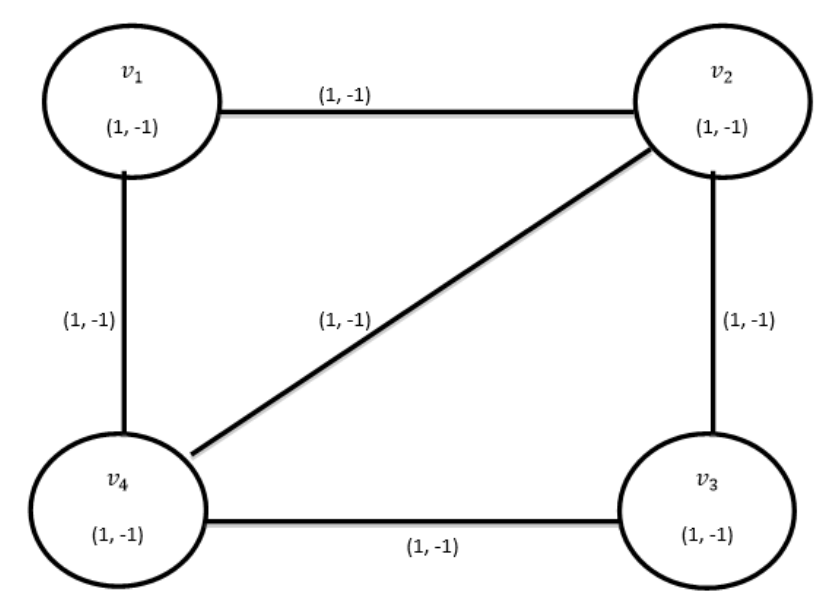

Figure 4: $\rho$-perfect bipolar fuzzy graph.

Proposition 3.9. Every $\rho$-perfect bipolar fuzzy graph is an $\mu$-perfect bipolar fuzzy graph.

Proof. Let $\mathrm{G}=(\mathrm{V}, \mathrm{E}, \mu, \rho)$ be a $\rho$-perfect bipolar fuzzy graph. Then all edges in $\mathrm{G}$ have the bipolar fuzzy values $(1,-1)$, i.e., $\rho(v w)=(1,-1), \forall v w \in E$. By the definition of bipolar fuzzy graph, we have $\rho(\nu w)=\left(\rho^{\mathrm{P}}(v w), \rho^{\mathrm{N}}(\nu w)\right)$, where $\rho^{\mathrm{P}}(\nu w) \leqslant \min \left(\mu^{\mathrm{P}}(v), \mu^{\mathrm{P}}(w)\right)$ and $\rho^{\mathrm{N}}(\nu w) \geqslant \max \left(\mu^{\mathrm{N}}(v), \mu^{\mathrm{N}}(w)\right)$. This implies that $1 \leqslant \min \left(\mu^{\mathrm{P}}(v), \mu^{\mathrm{P}}(w)\right)$ and $-1 \geqslant \max \left(\mu^{\mathrm{N}}(v), \mu^{\mathrm{N}}(w)\right)$. Further, this implies that 
$\mu^{\mathrm{P}}(v)=1, \mu^{\mathrm{P}}(w)=1$, because greater than 1 is not possible; similarly, $\mu^{\mathrm{N}}(v)=-1, \mu^{\mathrm{N}}(w)=-1$, because less than -1 is not possible. Therefore $\left(\mu^{\mathrm{P}}(v), \mu^{\mathrm{N}}(v)\right)=(1,-1)$ and $\left(\mu^{\mathrm{P}}(w), \mu^{\mathrm{N}}(w)\right)=(1,-1)$, that is, $\mu(v)=(1,-1)$ and $\mu(w)=(1,-1)$. In general, $\mu\left(v_{i}\right)=(1,-1), \forall v_{i} \in V$. Hence, every $\rho$-perfect bipolar fuzzy graph is an $\mu$-perfect bipolar fuzzy graph.

Note that the converse of the Proposition 3.9 need not be true. See Example 3.7.

Definition 3.10. A bipolar fuzzy graph $G=(V, E, \mu, \rho)$ is called a perfect bipolar fuzzy graph if it is an $\rho$-perfect bipolar fuzzy graph.

Definition 3.11. A bipolar fuzzy graph $G=(V, E, \mu, \rho)$ is called a complete perfect bipolar fuzzy graph if $\rho^{\mathrm{P}}(\nu w)=1$ and $\rho^{\mathrm{N}}(\nu w)=-1$, i.e., $\rho(\nu w)=(1,-1)$ for all $v, w \in \mathrm{V}$.

Definition 3.12. A bipolar fuzzy graph $G=(V, E, \mu, \rho)$ is called semi-perfect bipolar fuzzy graph if all vertices in $\mathrm{G}$ are $\mu$-perfect bipolar fuzzy vertices.

Proposition 3.13. Every complete perfect bipolar fuzzy graph is a perfect bipolar fuzzy graph.

Proof. Let $G=(V, E, \mu, \rho)$ be a complete perfect bipolar fuzzy graph. Since $G$ is complete, all vertices are connected together. Since $\mathrm{G}$ is perfect, all edges are $\rho$-perfect. That is, $\rho(v w)=(1,-1), \forall v w \in E$. Therefore, obviously $\mathrm{G}$ is a perfect bipolar fuzzy graph.

Note that every perfect bipolar fuzzy graph is not necessarily a complete perfect bipolar fuzzy graph.

Proposition 3.14. Every complete perfect bipolar fuzzy graph is a semi-perfect bipolar fuzzy graph.

Proof. Since $\mathrm{G}=(\mathrm{V}, \mathrm{E}, \mu, \rho)$ is complete perfect bipolar fuzzy graph, all edges are $\rho$-perfect and all vertices are joined by an edge. Clearly, $\mathrm{G}$ is $\mu$-perfect. Therefore, $\mathrm{G}$ is a semi-perfect. Hence, every complete perfect bipolar fuzzy graph is a semi-perfect bipolar fuzzy graph.

Note that the converse of the Proposition 3.14 need not be true.

Proposition 3.15. In a semi-perfect bipolar fuzzy graph, the following equations hold:

(i) $\sum_{v_{i}} \mathrm{~d}_{\mathrm{N}}\left(v_{\mathrm{i}}\right)=2|\mathrm{E}| \times(-1,1), \forall v_{\mathrm{i}} \in \mathrm{V}$;

(ii) $\sum_{v_{i}} d_{N}\left[v_{i}\right]=(2|E|+|V|) \times(-1,1), \forall v_{i} \in V$.

Proof. Since $G=(V, E, \mu, \rho)$ is semi-perfect bipolar fuzzy graph, it is $\mu$-perfect.

(i) By definition of neighborhood degree of any vertex in bipolar fuzzy graph $G$, we have $d_{N}\left(v_{i}\right)=$ $(m,-m)=m(1,-1)$, where $m$ is number of edges incidence with $v_{i}$, and every edge is connected by two vertices. Therefore, $\sum_{v_{i}} d_{N}\left(v_{i}\right)=2|E| \times(-1,1)$.

(ii) By definition of closed neighborhood degree of any vertex in $G$, we have $d_{N}\left[v_{i}\right]=(m,-m)+(1,-1)$, where $m$ is number of edges incidence with $v_{i}$, and every edge is connected by two vertices. Therefore, $\sum_{v_{i}} d_{N}\left[v_{i}\right]=2|E| \times(-1,1)+|V| \times(1,-1)=(2|E|+|V|) \times(1,-1)$.

Proposition 3.16. In a semi-perfect and semi-complete bipolar fuzzy graph, $\sum_{v_{i}} d_{N}\left[v_{i}\right]=|V| \times O(G)$.

Proof. Let $\mathrm{G}=(\mathrm{V}, \mathrm{E}, \mu, \rho)$ be a semi-perfect and semi-complete bipolar fuzzy graph with $n$ vertices, that is $|V|=n$ and $O(G)=(n,-n)$. Since $G=(V, E, \mu, \rho)$ is semi-perfect, it is $\mu$-perfect, the closed neighborhood degree of any vertex in $\mathrm{G}$ is $|\mathrm{V}| \times(1,-1)$, and by definition of semi-complete bipolar fuzzy graph, we have $\sum_{v_{i}} d_{N}\left[v_{i}\right]=(n,-n)+(n,-n)+\cdots+($ for $n$ vertices $)=\mathfrak{n} \times(\mathfrak{n},-\mathfrak{n})=|V| \times O(G)$.

Proposition 3.17. If $\mathrm{G}=(\mathrm{V}, \mathrm{E}, \mu, \rho)$ is a perfect bipolar fuzzy graph, then

(i) $\mathrm{d}\left(v_{\mathrm{i}}\right)=\mathrm{d}_{\mathrm{E}}\left(v_{\mathrm{i}}\right)=\mathrm{d}_{\mathrm{N}}\left(v_{\mathrm{i}}\right), \forall v_{\mathrm{i}} \in \mathrm{V}$;

(ii) $\sum_{v_{i}} \mathrm{~d}\left(v_{\mathrm{i}}\right)=\sum_{v_{i}} \mathrm{~d}_{\mathrm{E}}\left(v_{\mathrm{i}}\right)=\sum_{v_{i}} \mathrm{~d}_{\mathrm{N}}\left(v_{\mathrm{i}}\right), \forall v_{\mathrm{i}} \in \mathrm{V}$. 
Proof. Since $\mathrm{G}=(\mathrm{V}, \mathrm{E}, \mu, \rho)$ is perfect bipolar fuzzy graph, it has $\mu$-perfect and $\rho$-perfect, by definitions of degree, effective degree and neighborhood degree, the results are immediate.

Let us verify the Proposition 3.17 by the following example.

Example 3.18. Let $G=(V, E, \mu, \rho)$ be a perfect bipolar fuzzy graph, where $V=\left\{v_{1}, v_{2}, v_{3}, v_{4}, v_{5}\right\}$, and $E=$ $\left\{v_{1} v_{2}, v_{2} v_{3}, v_{2} v_{4}, v_{3} v_{4}, v_{3} v_{5}, v_{4} v_{5}\right\}$ with $\mu\left(v_{1}\right)=\mu\left(v_{2}\right)=\mu\left(v_{3}\right)=\mu\left(v_{4}\right)=\mu\left(v_{5}\right)=(1,-1) ; \rho\left(v_{1} v_{2}\right)=$ $\rho\left(v_{2} v_{3}\right)=\rho\left(v_{2} v_{4}\right)=\rho\left(v_{3} v_{4}\right)=\rho\left(v_{3} v_{5}\right)=\rho\left(v_{4} v_{5}\right)=(1,-1)$. This is an $\mu$-perfect and $\rho$-perfect bipolar fuzzy graph. By usual calculations, we get $\mathrm{d}\left(v_{1}\right)=\mathrm{d}_{\mathrm{E}}\left(v_{1}\right)=\mathrm{d}_{\mathrm{N}}\left(v_{1}\right)=(1,-1) ; \mathrm{d}\left(v_{2}\right)=\mathrm{d}_{\mathrm{E}}\left(v_{2}\right)=$ $\mathrm{d}_{\mathrm{N}}\left(v_{2}\right)=(3,-3) ; \mathrm{d}\left(v_{3}\right)=\mathrm{d}_{\mathrm{E}}\left(v_{3}\right)=\mathrm{d}_{\mathrm{N}}\left(v_{3}\right)=(3,-3) ; \mathrm{d}\left(v_{4}\right)=\mathrm{d}_{\mathrm{E}}\left(v_{4}\right)=\mathrm{d}_{\mathrm{N}}\left(v_{4}\right)=(3,-3) ; \mathrm{d}\left(v_{5}\right)=$ $\mathrm{d}_{\mathrm{E}}\left(v_{5}\right)=\mathrm{d}_{\mathrm{N}}\left(v_{5}\right)=(2,-2)$. In general, $\mathrm{d}\left(v_{\mathrm{i}}\right)=\mathrm{d}_{\mathrm{E}}\left(v_{\mathrm{i}}\right)=\mathrm{d}_{\mathrm{N}}\left(v_{i}\right), \forall v_{i} \in V$. Further, the sum of the degrees, sum of the effective degrees and sum of the neighborhood degrees are same, that is,

$$
\sum_{v_{i}} \mathrm{~d}\left(v_{\mathrm{i}}\right)=\sum_{v_{\mathrm{i}}} \mathrm{d}_{\mathrm{E}}\left(v_{\mathrm{i}}\right)=\sum_{\nu_{\mathrm{i}}} \mathrm{d}_{\mathrm{N}}\left(\nu_{\mathrm{i}}\right)=(12,-12), \forall v_{\mathrm{i}} \in \mathrm{V}
$$

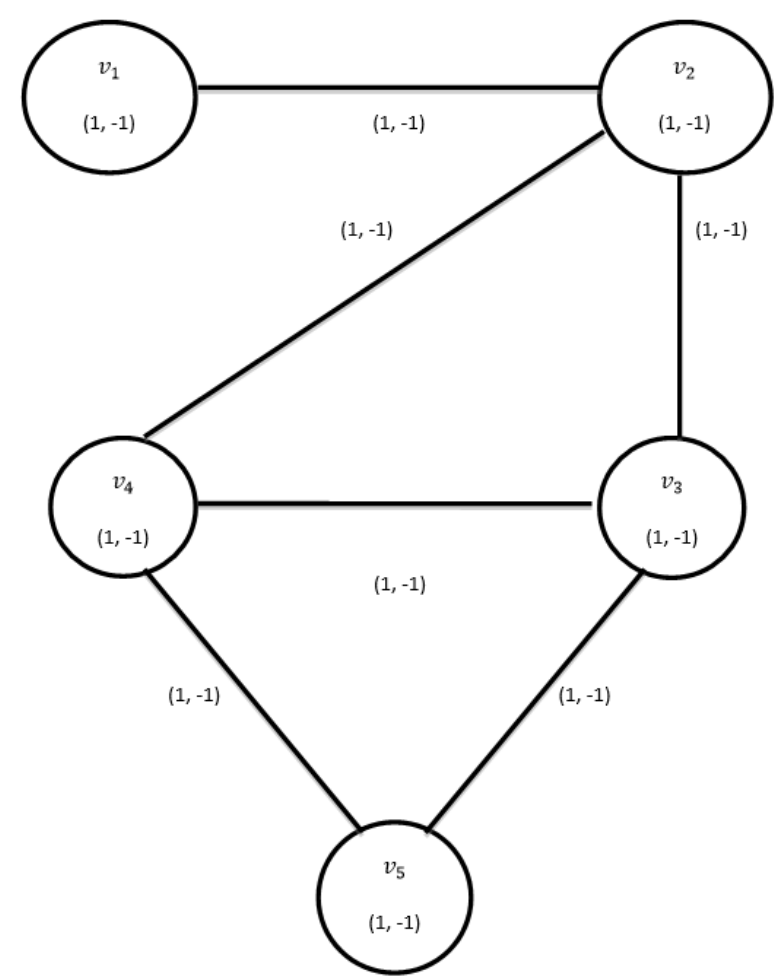

Figure 5: Perfect bipolar fuzzy graph.

Proposition 3.19. In any complete perfect bipolar fuzzy graph, $\mathrm{d}\left(v_{\mathrm{i}}\right)=\mathrm{d}_{\mathrm{E}}\left(v_{\mathrm{i}}\right)=\mathrm{d}_{\mathrm{N}}\left(v_{\mathrm{i}}\right), \forall v_{i} \in \mathrm{V}$.

Proof. Proof is obvious.

Proposition 3.20. In a complete perfect bipolar fuzzy graph,

(i) $\mathrm{O}(\mathrm{G})=\mathrm{d}_{\mathrm{N}}\left[v_{\mathrm{i}}\right], \forall v_{\mathrm{i}} \in \mathrm{V}$;

(ii) $\mathrm{S}(\mathrm{G})=\frac{(\mathrm{n}-1)}{2} \mathrm{O}(\mathrm{G})$, where $\mathrm{n}=|\mathrm{V}|$.

Proof. Let $\mathrm{G}=(\mathrm{V}, \mathrm{E}, \mu, \rho)$ be a complete perfect bipolar fuzzy graph with $n$ vertices, that is $|\mathrm{V}|=n$ and $\mathrm{O}(\mathrm{G})=(\mathrm{n},-\mathrm{n})$. 
(i) Since $G$ is complete perfect bipolar fuzzy graph with $n$ vertices, the closed neighborhood degree of any vertex is $(n-1,-(n-1))+(1,-1)=(n-1+1,-(n-1)-1)=(n,-n)$, i.e., $d_{N}\left[v_{i}\right]=(n,-n)=$ $\mathrm{O}(\mathrm{G}), \forall v_{\mathrm{i}} \in \mathrm{V}$.

(ii) Since $G$ is complete perfect bipolar fuzzy graph and by definition of size, $S(G)=\left(S^{P}(G), S^{N}(G)\right)=$ $\frac{\mathrm{n}(\mathrm{n}-1)}{2}(1,-1)=\frac{(\mathrm{n}-1)}{2}(\mathrm{n},-\mathrm{n})=\frac{(\mathrm{n}-1)}{2} \mathrm{O}(\mathrm{G})$.

Proposition 3.21. Every complete perfect bipolar fuzzy graph is regular bipolar fuzzy graph.

Proof. Since $\mathrm{G}$ is complete perfect bipolar fuzzy graph with $n$ vertices; the (open) neighborhood degree of any vertex is $(n-1,-(n-1))$, i.e., $d_{N}\left(v_{i}\right)=(n-1,-(n-1))=(n-1) \times(1,-1), \forall v_{i} \in V$. This is an $(n-1)$-regular bipolar fuzzy graph. Thus, every complete perfect bipolar fuzzy graph is regular bipolar fuzzy graph.

Proposition 3.22. Every semi-perfect bipolar fuzzy graph is not necessarily regular bipolar fuzzy graph.

Proof. Proof is straightforward.

\section{Conclusion}

This research article has introduced the perfect bipolar fuzzy graph, complete perfect bipolar fuzzy graph, and semi-perfect bipolar fuzzy graph. Further, it has given some properties related to those notions. In future, these ideas can be extended to other graph theoretical topics.

\section{References}

[1] M. Akram, Bipolar fuzzy graphs, Inform. Sci., 181 (2011), 5548-5564. 1, 2

[2] M. Akram, Bipolar fuzzy graphs with applications, Knowledge-Based Syst., 39 (2013), 1-8. 1

[3] M. Akram, W. A. Dudek, Regular bipolar fuzzy graphs, Neural Comput. Appl., 21 (2012), 197-205. 1

[4] M. Akram, N. Waseem, Novel applications of bipolar fuzzy graphs to decision making problems, J. Appl. Math. Comput., 56 (2018), 73-91.

[5] M. B. Ahamed, Types of degrees in bipolar fuzzy graphs, Appl. Math. Sci. (Ruse), 7 (2013), 4857-4866. 1

[6] M. B. Ahamed, A. Nagoor Gani, Perfect fuzzy graphs, Bull. Pure Appl. Sci., 28 (2009), 83-90. 1

[7] M. B. Ahamed, A. Nagoor Gani, Closed neighborhood degree and its extension in fuzzy graphs, Far East J. Appl. Math., 40 (2010), 65-72. 1

[8] P. Bhattacharya, Some remarks on fuzzy graphs, Pattern Recognit. Lett., 6 (1987), 297-302. 1

[9] K. R. Bhutani, A. Rosenfeld, Strong arcs in fuzzy graphs, Inform. Sci., 152 (2003), 319-322. 1, 2.2

[10] F. Harary, Graph Theory, Addison-Wesley, Reading, (1972).

[11] J. N. Mordeson, P. S. Nair, Fuzzy Graphs and Fuzzy Hypergraphs, Physica-Verlag, Heidelberg, (2000).

[12] A. Nagoor Gani, M. B. Ahamed, Order and Size in Fuzzy Graphs, Bull. Pure Appl. Sci. Sect. E Math. Stat., 22 (2003), $145-148$.

[13] A. Rosenfeld, Fuzzy graphs, in: Fuzzy sets and their applications to cognitive and decision processes, 1975 (1975), 77-95. 1

[14] L. A. Zadeh, Fuzzy sets, Inf. Control, 8 (1965), 338-353. 1

[15] J. Zhan, M. Akram, M. Sitara, Novel decision-making method based on bipolar neutrosophic information, Soft Computing, 2018 (2018), 1-23.

[16] W. R. Zhang, Bipolar fuzzy sets and relations: a computational framework for cognitive modeling and multiagent decision analysis, Proceedings of the First International Joint Conference of The North American Fuzzy Information Processing Society Biannual Conference, 1994 (1994), 305-309. 1, 2 